\title{
Decidim: redes políticas y tecnopolíticas para la democracia participativa
}

\author{
Decidim: political and technopolitical networks \\ for participatory democracy
}

XABIER BARANDIARAN, ${ }^{a}$ ANTONIO CALLEJA-LÓPEZ, ${ }^{\mathrm{b}}$ ARNAU MONTERDE, ${ }^{\mathrm{b}}$ PABLO ARAGÓN, ${ }^{\mathrm{c} *}$ JUAN LINARES, ${ }^{\mathrm{d}}{ }^{\mathrm{C}}$ AROL ROMERO ${ }^{\mathrm{e}}$ y ANDRÉS PEREIRA ${ }^{\mathrm{f}}$

${ }^{a}$ Upv/Ehu i AJuntament de Barcelona, ${ }^{\mathrm{b} U O C}$, ${ }^{\mathrm{c}}$ UPf I Eurecat, ${ }^{\mathrm{d}}$ UPf, ${ }^{\mathrm{e}}$ Consorci Localret, ${ }^{\mathrm{f}}$ Asociación alabs

Artículo recibido: 16 junio 2017

Artículo aceptado: 18 julio 2017

\section{INTRODUCCIÓN: DECIDIM Y EL CICLO TECNOPOLÍTICO 15M}

En 2011, año clave de una profunda crisis económica y política en España, dos lemas resonaron con fuerza en las calles y en las redes. El primero, crítico, denunciaba que los y las representantes políticos «no nos representan»; el segundo, utópico, apuntaba al deseo multitudinario de una «democracia real, ¡ya!». Estos lemas del denominado Movimiento 15M abrieron un ciclo político que ha situado en el centro del debate público la cuestión de la relación entre representación y democracia, y que ha encontrado en el uso intensivo y extensivo de las tecnologías de la información y la comunicación una vía para ir más allá de la primera a fin de reconstruir la segunda. Entre 2013 y 2015 este ciclo favoreció el surgimiento de nuevos partidos como Podemos y numerosas candidaturas municipalistas (de Barcelona en Comú a Ahora Madrid), iniciativas que han reformulado la política de partidos desde lógicas más participativas, abiertas y en red.Tras el ascenso de algunas de estas iniciativas a los gobiernos de diferentes ciudades, en 2015 y 2016, Madrid y Barcelona fueron pioneras en el desarrollo de plataformas digitales de democracia participativa que han abierto un nuevo periodo de innovación democrática. Decide Madrid y Decidim Barcelona (inicialmente basada en Cónsul, la aplicación base de Decide Madrid) son la punta de lanza de estas innovaciones. Con ellas, mu-

Pablo Aragón cuenta con el apoyo del Ministerio de Economía y Competitividad de España en el marco del Programa de Unidades de Excelencia María de Maeztu (MDM-2015-0502).

Pablo Aragón is supported by the Spanish Ministry of Economy and Competitiveness under the María de Maeztu Units of Excellence Programme (MDM-2015-0502). 
chos de los actores y reclamos del ciclo político abierto por el 15M alcanzaban las estructuras institucionales de la política pública. Lo hacían con el objetivo de reconstruirlas, de articular nuevos mecanismos más allá de la representación, orientados a construir una democracia más real.

\section{1. ¿QUÉ ES DECIDIM?}

Decidim $^{1}$ es una plataforma digital de participación política construida por el Ajuntament de Barcelona. Su versión más avanzada, desplegada en la ciudad de Barcelona, puede visitarse en https://decidim.barcelona, pero también en instancias ya activas en otros municipios e instituciones que comienzan a utilizarlo. Está basada en código libre (con una licencia GNU Affero General Public License v3.0), que puede leerse, comentarse, mejorarse y copiarse en la plataforma de programación colaborativa Gitbub. ${ }^{2}$

Más allá de su realidad como plataforma, Decidim es un complejo proyecto tecnopolítico cuyas múltiples dimensiones esbozamos en este artículo. El proyecto ha exigido y permitido ensamblar toda una serie de códigos que van más allá del informático y que, en conjunto (habida cuenta de su hibridación práctica de política y tecnología) denominamos «tecnopolíticos»: códigos jurídicos, institucionales, prácticos, sociales, discursivos, económicos e incluso filosóficos. Estos códigos ocupan roles diferentes pero igualmente cruciales en la construcción de una democracia en red (esto es, una democracia radicalmente mediada por las tecnologías de la información y la comunicación). En sentido amplio, Decidim es «una infraestructura abierta y libre para la democracia participativa», además de un proyecto que aspira a servir de dispositivo y modelo para la transformación política.

\section{DATOS, FUNCIONALIDADES Y CARACTERÍSTICAS GENERALES DE DECIDIM}

Decidim es un proyecto que nace en febrero de 2016. A día de hoy (20 de julio de 2017) se ha extendido a otras 9 ciudades (Hospitalet, Gavà, Badalona, Sabadell, Terrassa, Sant Cugat, Vilanova i la Geltrú, Mataró y Pamplona) y se encuentra en proceso de adopción por otras instituciones como la Di-

1 Más información en http://decidim.org.

2 Más información en https://github.com/decidim/decidim. 
putación de Barcelona o la Generalitat de Catalunya. ${ }^{3}$ Solo en Barcelona hay 26.700 personas registradas, se han abierto 12 procesos de participación y una iniciativa ciudadana, se han realizado un total de 11.873 propuestas, de las cuales más de un $70 \%$ han sido adoptadas como política pública por el ayuntamiento, recogidas en 1.678 actuaciones o resultados de procesos de participación cuyo seguimiento y monitorización es activo y transparente. A través de la plataforma se han generado cientos de miles de interacciones digitales (por ejemplo, 185.783 apoyos a propuestas, 19.126 comentarios y un número indeterminado de comparticiones en redes sociales).

Decidim cuenta con una serie de funcionalidades que han sido desplegadas con éxito y que incluyen: propuestas abiertas y apoyos a propuestas, encuentros presenciales (con convocatoria abierta, geolocalización y publicación de actas), debates abiertos con cargos electos, comentarios deliberativos y discusión de propuestas, actuaciones o resultados (que agrupan propuestas, detallan la aceptación o rechazo de las propuestas, y recogen los metadatos de encuentros, apoyos e interacciones), encuestas, iniciativas ciudadanas, varios sistemas de voto (incluyendo presupuestos participativos), protocolos de apertura y visualización de datos, seguimiento de resultados y rendición de cuentas, e integración con redes sociales. Estas funcionalidades se han combinado para realizar un abanico de procesos que incluyen presupuestos participativos, reglamentos abiertos, diseño colaborativo de software o planificación estratégica multiescala. Otras funcionalidades que ya están disponibles pero no han sido activadas todavía incluyen: procesos de elección de cargos, órganos de participación, debates abiertos y umbrales de filtrado colaborativo. Finalmente, entre las funcionalidades previstas para su desarrollo y despliegue antes de julio de 2018 se incluyen: definición participativa de orden del día para consejos, plenos y órganos de gobierno, legislación colaborativa, comunicación horizontal (privada y pública) entre usuarias, federación de servidores, integración con voto electrónico seguro, back-end distribuido, gamificación, app móvil y firmas digitales.

La traducción de los procesos tradicionales de participación a una plataforma digital ha implicado diseñar un configurador de procesos, una matriz abstracta para la generación de procesos a partir de componentes o elementos básicos. Decidim permite, a través de este configurador, crear cualquier tipo de proceso de participación. A continuación describimos someramente la estructura básica (u ontología) de Decidim a día de hoy, en la que se basa el mencionado configurador.

3 Para un mapa actualizado de implantación, consúltese https://decidim-monitor.herokuapp.com/. 


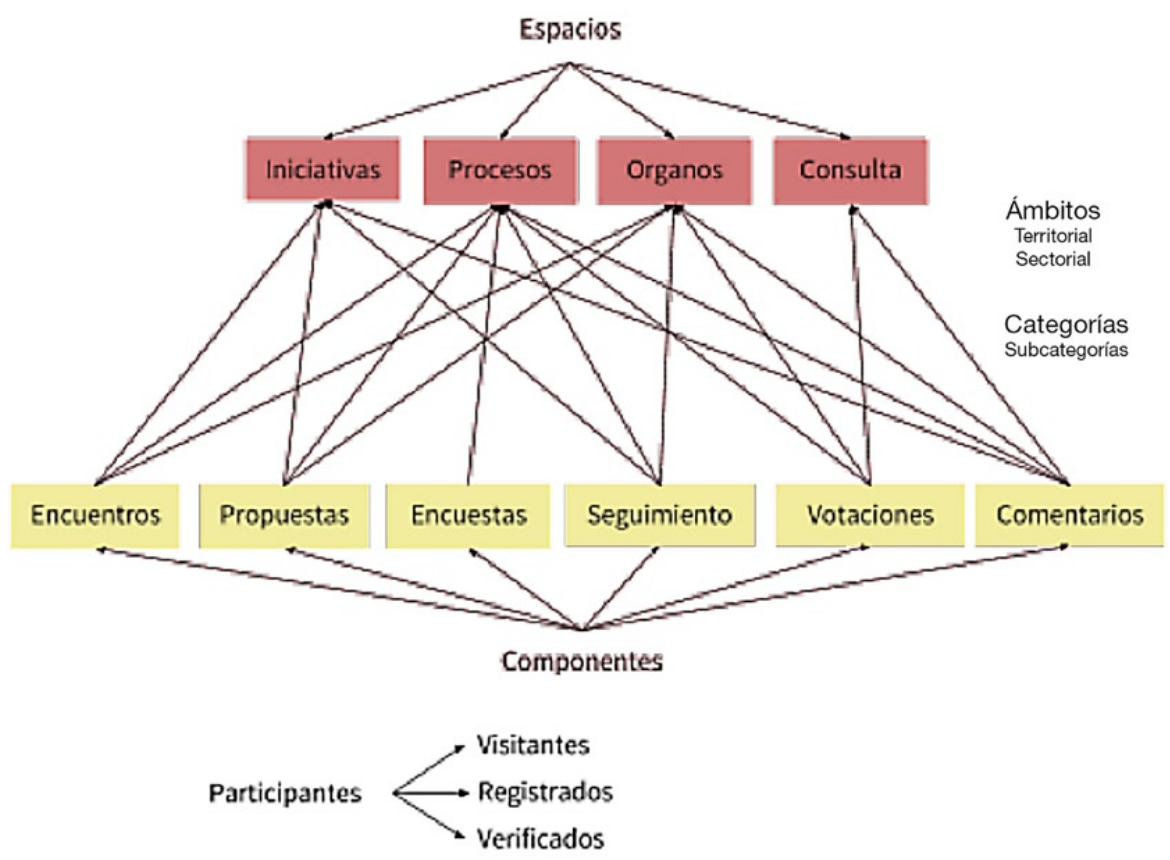

Figura 1

Diagrama de la ontología básica de Decidim

Las personas usuarias de la plataforma interactúan a través de componentes con funcionalidades específicas en espacios de participación. Dicho de otro modo, los espacios de participación como las Iniciativas, los Órganos, los Procesos o las Consultas disponen de componentes que se combinan como mecanismos de participación. Entre los componentes más destacados están los encuentros presenciales, las encuestas, las propuestas, las votaciones y los comentarios. Por ejemplo, un proceso de participación específico (como un proceso de presupuestos participativos) puede combinar en sus diferentes fases la posibilidad de convocar y registrar los resultados de encuentros presenciales con la ciudadanía, puede incluir una encuesta, la posibilidad de hacer propuestas, y después de un periodo de comentarios y debates sobre las propuestas, activar el componente de votación. Existen fundamentalmente tres tipos de participantes: visitantes 
(pueden acceder y leer el contenido de la plataforma), registrados (puede realizar comentarios y propuestas), y verificados (pueden votar o firmar). Cada instancia de un espacio de participación (por ejemplo, un proceso de participación) se sitúa dentro de un ámbito (territorial y/o sectorial) y se define por una serie de categorías y subcategorías (por ejemplo, temáticas) que afectan solo a dicho espacio.

A diferencia de otras plataformas existentes, la arquitectura de Decidim es modular, escalable y fácilmente configurable. No hace falta saber programar para instalar y utilizar la plataforma, que ha sido diseñada para facilitar la configuración y despliegue de procesos, órganos y mecanismos participativos desde un panel de administración. Los módulos que la integran son independientes entre sí y pueden ser desarrollados, activados y desactivados de manera independiente.

La gobernanza de Decidim se organiza alrededor de MetaDecidim: un espacio de encuentro, un proceso y una comunidad participativos, orientados a decidir, diseñar y producir de manera colaborativa y democrática múltiples aspectos del proyecto. Gracias al proceso de participación abierto en el portal decidim.barcelona, ${ }^{4}$ la posterior apertura de un portal propio en meta.decidim.barcelona, ${ }^{5}$ y los encuentros mensuales som, ${ }^{6}$ el propio Decidim sirve para el gobierno y el diseño democrático tanto del software como del proyecto en un sentido más amplio. La producción del software utiliza Github como plataforma colaborativa y los tickets (unidades de trabajo de software) se conectan con los procesos y espacios MetaDecidim.

El proyecto implica un partenariado público-común. Decidim es una infraestructura digital libre, abierta y democrática, cuyas funcionalidades nucleares y cuya sostenibilidad vienen aseguradas por fondos públicos y alrededor del cual se organizan una serie de agentes productivos y de servicios, tanto comunitarios, como públicos o privados. Decidim está mantenido y desarrollado por un ecosistema creciente compuesto por empresas, fundaciones, ciudadanía, asociaciones, hackers, personas investigadoras y diversas instituciones. Los servicios y recursos generados por este ecosistema van más allá de las necesidades institucionales públicas y atienden a las necesidades del tejido asociativo, cooperativo o colaborativo social, permitiendo la coordinación democrática a múltiples escalas de la inteligencia social.

4 Más información en https://www.decidim.barcelona/processes/12.

5 Más información en https://meta.decidim.barcelona/.

6 Más información en https://www.decidim.barcelona/processes/12/f/47/?locale=es. 
A modo de contrato sociotécnico, un código legal de garantías democráticas vincula a la comunidad y a sus usuarias, sean personas o instituciones. Han de respetarse una serie de principios de implementación, uso y desarrollo: principio de colaboración; principio de transparencia, trazabilidad e integridad de la participación; principio de privacidad; principio de nodiscriminación e igualdad de oportunidades entre personas y entre propuestas; principio de inclusividad y principio de participación multicapa. ${ }^{7}$

\section{USOS DE DECIDIM: EL CASO DEL PAM 2016-2019}

Hasta la fecha, el proceso de participación de mayor impacto político, administrativo y social ha sido el que llevó al desarrollo conjunto del PAM (Plan de Actuación Municipal) y los PAD (Plan de Actuación de Distritos). Este plan define las líneas estratégicas de actuación del gobierno municipal durante el mandato. A continuación, resumimos brevemente algunas claves del proceso de participación del PAM y proporcionamos una serie de datos, tales como los indicadores de la participación en el proceso, así como algunas visualizaciones sobre los contenidos, las redes de participación y la distribución geográfica de los diferentes encuentros presenciales.

\subsection{Desarrollo del proceso}

El proceso participativo para la elaboración del PAM se inició en el mes de noviembre de 2015 y se dividió en varias fases. Aunque hubo participación en casi todas las fases del proceso, esta se concentró en la fase de recogida de propuestas ciudadanas, que se llevó a cabo entre el 1 de febrero y el 9 de abril de 2016, tanto en las encuentros presenciales (debates, jornadas y sesiones en órganos de participación), como a través de Decidim Barcelona.

7 El código de garantías democráticas es accesible en https://github.com/AjuntamentdeBarcelona/ decidim-doc-plandedesarrollo/blob/master/anexo-2.-codigo-de-garantias-democraticas-ycolaboracion-abierta.md. 


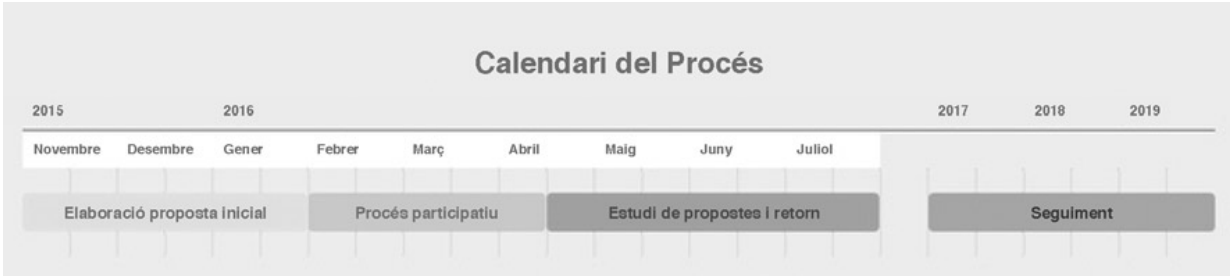

Figura 2

Calendario del proceso participativo del pam (2016-2019)

\subsection{Fase de elaboración de la propuesta inicial}

En esta fase, el ayuntamiento definió los ejes temáticos y las líneas de actuación derivadas de cada eje en que se dividían las diferentes propuestas de actuación del plan estratégico: buen vivir, transición ecológica, economía plural, buen gobierno y justicia global. Esta misma estructura se reprodujo para cada uno de los 10 distritos de la ciudad, obteniendo un documento propuesta para toda la ciudad (PAM) y otro para cada uno de los distritos (PAD). Esto resultó en la primera versión del PAM, de donde salieron las propuestas de actuación presentadas inicialmente por el ayuntamiento, que después pasaron a la fase de recogida de propuestas.

\section{3. fase de recogida, debate y priorización de propuestas}

En esta fase se habilitó un espacio en Decidim Barcelona donde se volcaron las propuestas iniciales del ayuntamiento, donde la ciudadanía podía tanto navegar por la propuesta inicial del consistorio como realizar nuevas propuestas enmarcándolas en los diferentes ejes y líneas de actuación definidas previamente. Cualquier persona usuaria de Decidim podía presentar nuevas propuestas y comentarlas proporcionando argumentos a favor y en contra, así como votar los comentarios. Sin embargo, solo personas empadronadas en la ciudad podían emitir apoyos a las propuestas. Durante este fase se realizaron encuentros presenciales (jornadas de ciudad y encuentros presenciales) donde se discutieron y recogieron las diferentes propuestas de manera presencial. Los encuentros fueron documentados y recogidas en la plataforma, y las propuestas surgidas fueron también transferidas a ella, incorporándose así al flujo de propuestas de Decidim Barce- 
lona. Finalmente, se realizaron una serie de debates digitales con la alcaldesa y los diferentes tenientes de alcalde para dar respuestas a la ciudadanía, relacionadas con las propuestas planteadas por el ayuntamiento en el documento inicial.

\subsection{Fase de estudio de propuestas y retorno}

En esta fase, las diferentes propuestas ciudadanas fueron evaluadas por los responsables de las diferentes áreas y distritos del ayuntamiento y sus equipos, proceso que involucró a más de un centenar de personas. Se estudiaron las propuestas teniendo en cuenta sus apoyos y comentarios, los debates presenciales en los que se habían discutido, las organizaciones involucradas en la propuesta, su viabilidad temporal, presupuestaria y técnica, así como su coherencia con las competencias municipales. Se estudiaron todas las propuestas, se agruparon las similares, y se rechazaron aquellas que no cumplían los criterios planteados. Todas las propuestas recibieron una respuesta pública en la plataforma respecto a su aceptación, modificación y agregación a una actuación, o su rechazo.

\subsection{Fase de alegaciones}

Una vez recogidas las propuestas y agrupadas en actuaciones se elaboró una nueva propuesta de Plan de Actuación Municipal (de ciudad y de distritos) como resultado del proceso participativo. Esta propuesta fue luego expuesta públicamente para recoger alegaciones según la legislación vigente. Una vez finalizado el proceso de recogida de alegaciones, el gobierno estudió e incorporó o desestimó cada una de las alegaciones en un nuevo documento. Las alegaciones vinculadas a las actuaciones concretas también quedaron recogidas en Decidim Barcelona.

\subsection{Aprobación final}

Este documento se llevó al Pleno Municipal, donde necesitaba una mayoría simple para ser aprobado. Los partidos de la oposición votaron en contra de dicha aprobación y, finalmente, el PAM no fue tramitado. 


\subsection{Fase de seguimiento}

Sin embargo, por la estructura de la administración municipal, el gobierno pudo avanzar con la implementación de las medidas del pam de manera desagregada. A tal efecto Decidim cuenta con un módulo de seguimiento que permitiría seguir y confirmar el grado de implementación de las actuaciones a lo largo del mandato.

\subsection{Resumen del proceso en decidim barcelona}

El proceso de elaboración colaborativa del pam contó con 39.049 personas participantes, 24.028 de las cuales lo hicieron a distancia y 15.021 de forma presencial. 1.741 organizaciones se involucraron en el proceso.

Tabla 1

Participantes del proceso participativo

\begin{tabular}{cc}
\hline Participantes en el proceso & 39.049 \\
\hline A distancia & 24.028 \\
Presencial & 15.021 \\
\hline
\end{tabular}

Tabla 2

Organizaciones participantes del proceso

\begin{tabular}{cc}
\hline $\begin{array}{c}\text { Organizaciones que se han dado } \\
\text { de alta en la plataforma }\end{array}$ & $\begin{array}{c}\text { Organizaciones que han participado } \\
\text { en encuentros presenciales }\end{array}$ \\
\hline 339 & 1.494 \\
\hline
\end{tabular}

La web decidim.barcelona recibió más de 247.000 visitas y se generaron 207.971 interacciones en línea con propuestas (apoyos a propuestas, comentarios y apoyos a comentarios). En cuanto a las formas de participación podemos diferenciar entre aportaciones ciudadanas, interacciones y espacios de participación. Las aportaciones se refieren al conjunto de in- 
tervenciones realizadas por las personas participantes en el proceso. Las interacciones miden el conjunto de acciones realizadas a la hora de participar en el mismo. Finalmente, se establecen los diferentes espacios abiertos de participación.

Tabla 3

Interacciones totales

\begin{tabular}{cccc}
\hline Propuestas & Comentarios & $\begin{array}{c}\text { Intervenciones en } \\
\text { encuentros }\end{array}$ & Apoyos \\
\hline 10.860 & 18.191 & 13.614 & 165.121 \\
\hline $\begin{array}{c}\text { Votos } \\
\text { en comentarios }\end{array}$ & $\begin{array}{c}\text { Debates } \\
\text { online }\end{array}$ & $\begin{array}{c}\text { Interacciones en los } \\
\text { puntos móviles }\end{array}$ & Total de interacciones \\
\hline 13.210 & 438 & 9.241 & 230.675 \\
\hline
\end{tabular}

Tabla 4

Acciones en los diferentes espacios de participación

\begin{tabular}{cccc}
\hline \multicolumn{3}{c}{ decidim.barcelona } \\
\hline Reuniones previas & Propuestas & Encuentros & Debates on line \\
138 & 10.860 & 410 & 5 \\
\hline
\end{tabular}

A continuación, se muestra el gráfico de interacciones entre usuarias de Decidim Barcelona. Cada nodo es una persona usuaria u organización de Decidim y cada enlace es una relación de respuesta en forma de comentario a una propuesta. De esta manera, se ilustra la complejidad de la red de conversación colectiva generada en torno a las propuestas del Plan Municipal. Se visualiza el papel central del Ayuntamiento de Barcelona, que presentó 1.300 propuestas iniciales, así como el de otros nodos y subredes que juegan un papel bastante importante en el tejido de interacciones. 

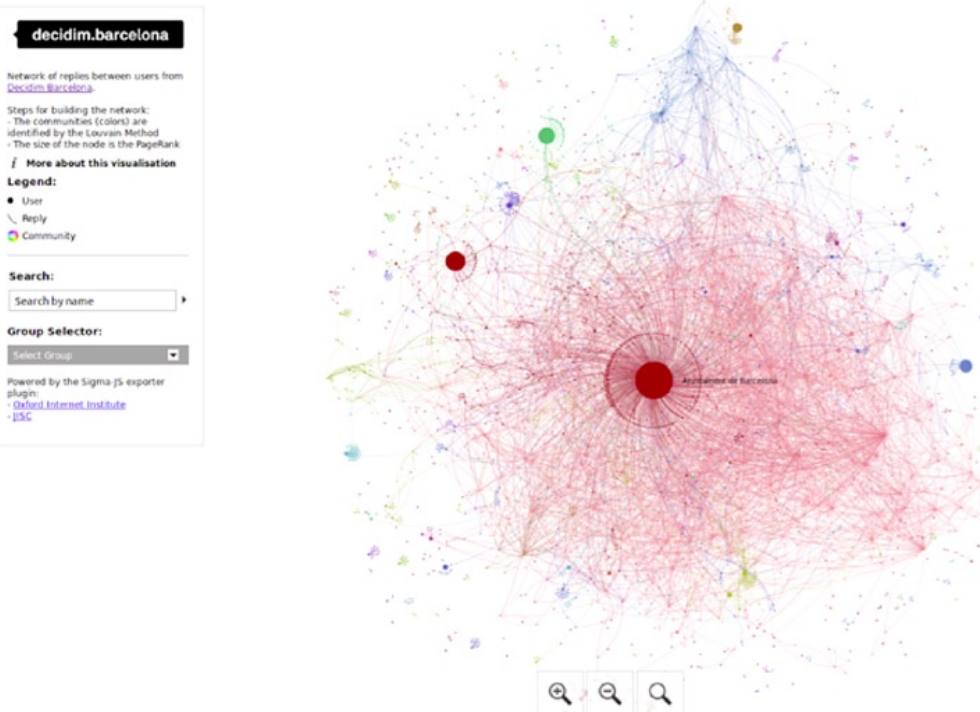

Figura 3

Red de interacción (respuestas) entre usuarios en el Decidim Barcelona. Más datos disponilbes en: http://decidim.barcelona/dades

Durante la fase previa de redacción de la primera versión del PAM se realizaron 138 reuniones presenciales, durante el proceso mismo se llevaron a cabo 410 citas o encuentros presenciales que fueron anunciados, registrados y documentados en Decidim Barcelona, así como 197 salidas de carretillas móviles, a través de las que podían realizarse propuestas. Los detalles de estos encuentros (actas, propuestas recogidas, número de participantes e intervenciones) fueron subidos a la plataforma, generando una dinámica multicapa entre la capa física y la capa digital. 
Tabla 5

Encuentros presenciales, participantes, intervenciones y propuestas según escala territorial

\begin{tabular}{lrrrr}
\hline \multicolumn{1}{c}{$\begin{array}{c}\text { Ámbito } \\
\text { territorial }\end{array}$} & Participaciones & Organizaciones & Intervenciones & $\begin{array}{c}\text { Propuestas } \\
\text { surgidas }\end{array}$ \\
\hline Ámbito ciudad & 3.860 & 872 & 5.425 & 1.942 \\
Ciutat Vella & 1.331 & 98 & 1.086 & 509 \\
Eixample & 906 & 112 & 740 & 308 \\
Sants-Montjüic & 622 & 153 & 1.072 & 386 \\
Les Corts & 415 & 15 & 250 & 183 \\
Sarrià-Sant Gervasi & 296 & 85 & 442 & 217 \\
Gràcia & 947 & 286 & 880 & 415 \\
Horta-Guinardó & 424 & 4 & 326 & 179 \\
Nou Barris & 1.488 & 90 & 1.807 & 219 \\
Sant Andreu & 611 & 127 & 495 & 382 \\
Sant Martí & 677 & 163 & 1.091 & 439 \\
\hline Total & 11.577 & 2.005 & 13.614 & 5.179 \\
\hline
\end{tabular}

Finalmente, con respecto a los impactos del proceso, puede apuntarse el alto porcentaje de propuestas ciudadanas seleccionadas (7.231 propuestas, $76,6 \%$ del total) para ser incorporadas, en forma de actuaciones, en la versión final del pam defendida por el equipo de gobierno en el pleno municipal. Hasta un $85,4 \%$ de las 8.470 propuestas incorporadas como actuaciones en la versión del pam propuesta por el gobierno resultan de ideas y propuestas ciudadanas. 


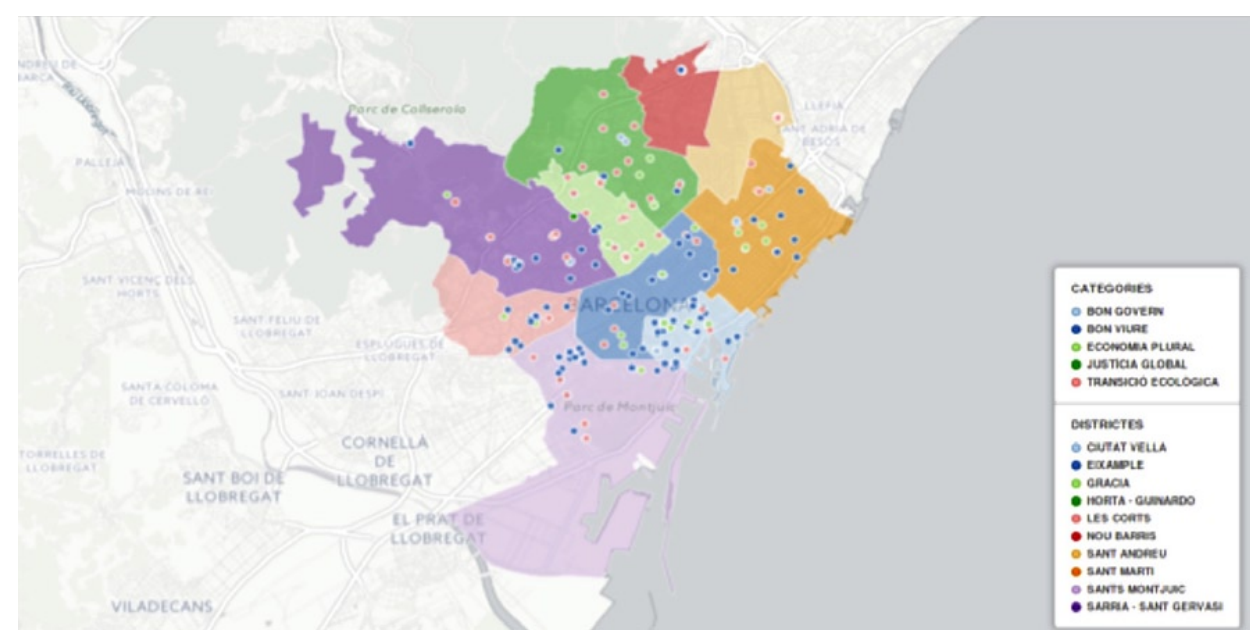

Figura 4

Mapa de encuentros presenciales, por ejes temáticos y distritos. Imagen y datos accesibles en: https://decidim.barcelona/dataviz/map

\section{CONCLUSIONES: REDES POLÍTICAS Y TECNOPOLÍTICAS PARA LA DEMOCRACIA DEL SIGLO XXI}

A pesar de la magnitud de los datos mencionados, entre los retos de futuro para el proyecto Decidim se encuentran el desarrollo de un mayor número de procesos vinculantes, una mayor distribución del poder público ligada a estructuras procomunales, o la reapropiación de Decidim entre colectivos sociales y cooperativas para la construcción de su autogobierno democrático y la ampliación de su capacidad de acción. Dicho esto, es importante subrayar que Decidim es una red digital de tercera generación (tras las redes informacionales predominantes en los 90 y las redes sociales de la última década), que comparte muchas características con ellas al tiempo que trata de evitar muchos de sus déficits. Decidim es ejemplo de un modelo emergente de redes digitales que denominamos «redes políticas». Estas redes tienen tres características fundamentales: en primer lugar, reducen la centralidad de la figura del prosumidor social y la sustituyen por la de un actor decididamente político; en segundo lugar, lo hacen articulando espacios que permiten la construcción de identidades, voluntades e inteligencias colectivas más allá de la mera expresión, agregación o cir- 
culación de gustos y preferencias individuales; en tercer lugar, conectan estas con decisiones que afectan al plano de lo colectivo en tanto que colectivo. En este sentido, las diferencias de nomenclatura son indicativas: en lugar de un Facebook (traducible como «libro de caras»), Decidim («decidimos», en castellano) sitúa en el centro el vínculo político, habla de un «nosotros y nosotras», un «nosotros y nosotras» decisivo, cuyo principio regulativo es el de que sus miembros «tomen parte como pares», en nuestra lectura del pars capere de la participación. Del mismo modo que las redes sociales construyeron sobre y, al tiempo, cuestionaron el modelo de las redes informacionales (conforme a la habitual reconstrucción del tránsito de la web 1.0 a la web 2.0), las redes políticas construyen sobre, conectan con y divergen de la lógica de las redes sociales.

Quizá de modo aún más interesante, más allá de su condición de red política, Decidim es un proyecto no superficial sino radicalmente participativo, esto es, permite el control y la intervención de las usuarias-participantes en todas las capas de su estructura, desde sus contenidos hasta su código. Esto lo distancia aún más del modelo habitual de red social corporativa, en el que las personas usuarias no tienen capacidad de decisión sobre aspectos como el código, las normas de uso o las políticas de datos. En este sentido, podríamos sugerir que el espacio y la comunidad MetaDecidim, mencionados en el apartado 2 , constituyen una red ya no solo política sino tecnopolítica, esto es, una red que sitúa la construcción de sus tecnologías en el centro de su acción política.

En esta crisis de la democracia representativa (neo)liberal, en la que las redes sociales han pasado a ocupar un rol central en la construcción de la política y de una emergente democracia en red, construir redes políticas y tecnopolíticas radicalmente democráticas e inteligentes (esto es, que movilicen lo que el 15M denominó «inteligencia colectiva») se convierte en un reto fundamental. En esta coyuntura, Decidim aspira a ser un proyecto y una infraestructura público-común, abierta y libre para la democracia participativa del siglo XXI. Un paso más allá de la representación, hacia la democracia real. 